\title{
Complexity in synchronized and non-synchronized states: a comparative analysis and application
}

\begin{abstract}
This analysis shows the dynamics of a hyperchaotic system changes from its original state to a synchronized state with nonlinear controller. The decreasing complexity of the coupled systems also quantifies the loss of information from its original state to the synchronized state. We proposed and modified a chaos synchronization based secure communication scheme to implement in case of non synchronization. The scheme is designed and illustrated using examples and simulations. Security analysis of the proposed scheme is also investigated. This analysis gives a new direction on chaos based cryptography in case of the coupled systems completely in non synchronized state.
\end{abstract}

